\title{
Degenerations of Algebras and Ranks of Grothendieck Groups
}

\section{Adam Hajduk}

Received: 5 July 2011 / Accepted: 17 September 2012 / Published online: 16 October 2012

(C) The Author(s) 2012. This article is published with open access at Springerlink.com

\begin{abstract}
A basic algebra $A_{0}$ is an idempotent rigid degeneration of a basic algebra $A_{1}$ if and only if it is a classical degeneration of $A_{1}$ and additionally $\operatorname{rk~} \mathrm{K}_{0}\left(A_{0}\right)=$ $\operatorname{rk~} \mathrm{K}_{0}\left(A_{1}\right)$.
\end{abstract}

Keywords Algebra • Locally bounded category • Algebraic variety • Degeneration of algebras $\cdot$ Degeneration order

Mathematics Subject Classifications (2010) $14 \mathrm{~A} 10 \cdot 16 \mathrm{G} 99 \cdot 06 \mathrm{~A} 06$

\section{Introduction}

Geometric degenerations of algebras were introduced more or less one hundred years ago. This notion is connected with considerations concerning affine algebraic variety $\operatorname{alg}_{d}(k)$ of the so called structure constants of $d$-dimensional $k$-algebras, which can be also interpreted as a set of all algebra structures on a $k$ linear space $k^{d}$. The group $\mathrm{Gl}_{d}(k)$ operates on $\operatorname{alg}_{d}(k)$ by the "base change" action in such a way that $\mathrm{Gl}_{d}(k)$-orbits correspond to isoclasses of $d$-dimensional algebras, and in this way we obtain degeneration order on the isoclasses of algebras. For

Presented by Idun Reiten.

Supported by the Research Grant N N201 542138 of the Polish Ministry of Science and Higher Education.

A. Hajduk $(\varangle)$

Faculty of Mathematics and Computer Science, Nicolaus Copernicus University,

Chopina 12/18, 87-100 Toruń, Poland

e-mail: ahajduk@mat.umk.pl 
any $d$-dimensional $k$-algebras $A_{0}, A_{1}$ with ordered bases $\bar{e}_{0}, \bar{e}_{1}$, we say that $A_{0}$ is a geometric degeneration of $A_{1}$, if the inclusion $\left.\mathrm{Gl}_{d}(k) \cdot c\left(A_{0}, \bar{e}_{0}\right) \subseteq \overline{\mathrm{Gl}_{d}(k) \cdot c\left(A_{1}, \bar{e}_{1}\right.}\right)$ holds, where $c(A, \bar{e})$ denotes the structure constants of $A$ with respect to $\bar{e}$ (see [3] for the precise definitions).

There is also a notion of a degeneration of finite locally bounded categories, which refers to the action of the smaller, closed subgroup $\mathrm{G}_{\underline{d}}(k)=\prod_{i, j=1}^{n} \mathrm{Gl}_{d_{i, j}}(k) \subseteq \mathrm{Gl}_{d}(k)$ on the subvariety $\operatorname{lbc}_{\underline{d}}(k) \subseteq \operatorname{alg}_{d}(k)$ consisting of locally bounded category structures with dimension vector $\underline{d} \in \mathbb{N}^{n^{2}}$, where $n \geq 1$ and $d:=n+\sum_{i, j=1}^{n} d_{i, j}$. This action is compatible with the previous one, hence the so called idempotent rigid degenerations of algebras form a subclass of classical degenerations (roughly speaking they are radical degenerations of locally bounded categories with the same dimension vector with respect to action of $\mathrm{G}_{\underline{d}}(k)$ on $\mathrm{lbc}_{\underline{d}}(k)$, see [3] for the precise definition).

A certain class of important problems concerning algebra degenerations naturally arises: we ask whether given a general property "P" is preserved under the process of algebra degeneration, i.e. if for an algebra $A_{1}$ with property "P" any degeneration $A_{0}$ of $A_{1}$ satisfies "P". For example, C. Geiss proved in [7], that any degeneration of a wild algebra is a wild algebra (see also [5] for infinite representation type). The representation types are one of the most useful properties preserved by algebra degenerations. There are of course also interesting properties "P" which are not preserved under the algebra degeneration process. In such case it is interesting to describe the subclass of all algebra degenerations for which property "P" is preserved.

In this paper we focus on the problem, having just such a character. We give a precise answer to the question: which algebra degenerations preserve the rank of Grothendieck group of an algebra, in case of basic algebras.

Note that idempotent rigid degenerations clearly preserve ranks of the Grothendieck group (they are also classical degenerations!). However, the converse is not true in general, even for $d=2$ (the semisimple $k$-algebra $k \times k$ degenerates to the local algebra $\left.k[T] /\left(T^{2}\right)\right)$. We characterize the class of all classical algebra degenerations, which can be realized as idempotent rigid ones, and show, that for basic algebras it holds in the most general possible case as it can be. Namely, if an algebra $A_{1}$ and its degeneration $A_{0}$ are basic, moreover, their Grothendieck groups have the same rank, then $A_{0}$ is a idempotent rigid degeneration of $A_{1}$ (see Theorem 3.1). As a conclusion, we infer that degenerations of basic algebras, which do not change the rank of Grothendieck group, are exactly the idempotent rigid ones.

The crucial role in the proof of our result is played by the notion of the so called generalized CB-degeneration of algebras (see [9] for a definition), which extends in some sense the notion of algebra degeneration introduced by W. Crawley-Boevey in [2]. The main idea relies on the realization of an algebra degeneration as a generalized CB-degeneration by some $k[X]$-algebra $\mathcal{A}$ in the best possible way (see Theorem 2.2), such that after some localization $\mathcal{A}$ comes from a concrete locally bounded $k[X]$-category $\mathcal{R}$, by taking corresponding $k[X]$-algebra $A(\mathcal{R})$. Now, using the characterization [4, Lemma 2.2] of idempotent rigid degenerations of algebras in terms of locally bounded $k[X]$-categories, we infer that $A_{0}$ is an idempotent rigid degeneration of $A_{1}$.

We use in the paper the classical notions and notation (for more specialized details see $[3,4,9])$. For information concerning basic facts in algebra and algebraic geometry used in this paper we refer to [10-13]. 


\section{Preliminaries, Notation, Basic Facts}

Throughout the paper $k$ will always denote an algebraically closed field, $X$ usually denotes a nonsingular affine curve, $U \subset X$ is a nonempty, open subset, $x_{0} \in X \backslash U$ and $x_{1} \in U$ are fixed points. For any $k$-algebra $A$ by $J(A)$ we denote the Jacobson radical of $A$. By $k[X]$ we denote the $k$-algebra of regular functions on $X$.

We recall briefly the notion of $k[X]$-algebra. For us it is just a $k$-algebra $\mathcal{A}$ on which $k[X]$ acts centrally.

For any $d \in \mathbb{N}$ we define the affine variety

$$
\operatorname{alg}_{d}(k):=\left\{c=\left(c_{i j}^{l}\right) \in k^{d^{3}} ;{ }_{c} \text { induces a } k \text {-algebra structure on } k^{d}\right\},
$$

where ${ }_{c}$ is the bilinear multiplication defined on the standard basis $e_{1}, \ldots, e_{d}$ of $k^{d}$ by the formula:

$$
e_{i} \cdot{ }_{c} e_{j}:=\sum_{l=1}^{d} c_{i j}^{l} \cdot e_{l},
$$

for $i, j=1, \ldots, n$. On this variety acts the full linear group $\mathrm{Gl}_{d}(k)$, this action on the algebra level corresponds to the transporting of an algebraic structure. Similarly, we can define the variety $\mathrm{lbc}_{\underline{d}}(k)$ of locally bounded categories with a fixed dimension vector $\underline{d} \in \mathbb{N}^{n^{2}}$ ( $n>0$ is also fixed). On this variety acts the smaller group $\mathrm{G}_{d}(k):=$ $\prod_{i, j=1}^{n} \overline{G l}_{d_{i, j}}(k)$. We can treat $\operatorname{lbc}_{\underline{d}}(k)$ as a closed subvariety of variety $\operatorname{alg}_{d}(k)$, where $d:=n+\sum_{i, j=1}^{n} d_{i, j}$ is a total dimension of the dimension vector $\underline{d}$. Moreover, these two actions are compatible under the standard embedding $\mathrm{G}_{d}(k) \hookrightarrow \mathrm{Gl}_{d}(k)$. It is clear that the orbits of the respective actions correspond to isoclasses of $d$ dimensional $k$-algebras (resp. isoclasses of ordered locally bounded $k$-categories with dimension vector $\underline{d}$ respectively), and any $d$-dimensional $k$-algebra $A$, under a choice of an ordered base, is uniquely represented by some element $c=c(A) \in \operatorname{alg}_{d}(k)$ (analogously for ordered locally bounded categories with the dimension vector $\underline{d}$, see [3] for details).

Definition Let $A_{0}$ and $A_{1}$ be finite dimensional algebras, with $\operatorname{dim}_{k} A_{0}=$ $\operatorname{dim}_{k} A_{1}=d$. We say that $A_{0}$ is a classical geometric degeneration of a $A_{1}$, if for structure constants $c_{0}, c_{1} \in \operatorname{alg}_{d}(k)$ corresponding to $A_{0}$ and $A_{1}$ respectively, the inclusion $\mathrm{Gl}_{d}(k) \cdot c_{0} \subseteq \overline{\mathrm{Gl}_{d}(k) \cdot c_{1}}$ holds, where the closure is taken in alg $(k)$. We say that $A_{0}$ is an idempotent rigid degeneration of $A_{1}$, if there exist $n>0$, a dimension vector $\underline{d} \in \mathbb{N}^{n^{2}}$ and ordered locally bounded $k$-categories $R_{0}, R_{1}$ with the dimension vector $\underline{d}$ such that $A\left(R_{0}\right) \cong A_{0}, A\left(R_{1}\right) \cong A_{1}$, and for the structure constants $c_{0}, c_{1} \in$ $\mathrm{lbc}_{\underline{d}}(k)$ corresponding to $R_{0}$ and $R_{1}$ respectively, the inclusion $\mathrm{G}_{\underline{d}}(k) \cdot c_{0} \subseteq \overline{\mathrm{G}_{\underline{d}}(k) \cdot c_{1}}$ holds, where the closure is taken in $\operatorname{lbc}_{d}(k)$ and $A\left(R_{i}\right)$ are the algebras associated to the categories $R_{i}$, for $i=0,1$.

We use the following notion: we write $A_{1} \leq_{\mathrm{cl}} A_{0}$ in case $A_{0}$ is a classical degeneration of $A_{1}$, and $A_{1} \leq$ rig $A_{0}$ in case $A_{0}$ is an idempotent rigid degeneration of $A_{1}$. 
For more precise definitions of classical and idempotent rigid degenerations and equivalent conditions we refer to [3].

\section{2}

For any $k[X]$-algebra $\mathcal{A}$ (resp. $k[X]$-module $\mathcal{M}$ ) and open subset $V \subseteq X$ by $\mathcal{A}_{V}$ (resp. $\mathcal{M}_{V}$ ) we mean the localization of $\mathcal{A}$ (resp. $\mathcal{M}$ ) to the open set $V$, i.e. $k[V]$-algebra $\mathcal{A}_{V}:=k[V] \otimes_{k[X]} \mathcal{A}$ (resp. $k[V]$-module $\mathcal{M}_{V}:=k[V] \otimes_{k[X]} \mathcal{M}$ ). We briefly recall, that for finite dimensional algebras $A_{0}$ and $A_{1}$, the algebra $A_{0}$ is called a generalized $C B$-degeneration of $A_{1}$, if there exist an affine variety $X$ with point $x_{0} \in X$, an open dense subset $U \subseteq X$ and a $k[X]$-algebra $\mathcal{A}$, which is finitely generated as a $k[X]$-module, such that $\mathcal{A}^{x_{0}} \cong A_{0}$ and $\mathcal{A}^{x} \cong A_{1}$ for $x \in U$, where $\mathcal{A}^{x}:=k[X] / \mathfrak{m}_{x} \otimes_{k[X]} \mathcal{A}$ (we refer to [9] for definitions and more information). The following result yields an useful equivalent condition which allows to find out that a $k$-algebra $A_{0}$ is a classical degeneration of a $k$-algebra $A_{1}$.

Theorem If $A_{1} \leq_{c l} A_{0}$, then there exist a nonsingular affine curve $X, x_{0}, x_{1} \in X, a$ $k[X]$-algebra $\mathcal{A}$, which is free as $k[X]$-module with $k[X]$-basis $v_{1}, \ldots, v_{d} \in \mathcal{A}$, and a regular morphism $g: U \rightarrow \mathrm{Gl}_{d}(k)$ such that:

(1) $\mathcal{A}^{x_{0}} \cong A_{0}$,

(2) $\mathcal{A}^{x} \cong A_{1}$, for all $x \in U$,

(3) $c\left(\mathcal{A}^{x},\left(v_{1}^{x}, \ldots, v_{d}^{x}\right)\right)=g(x) \cdot c\left(\mathcal{A}^{x_{1}},\left(v_{1}^{x_{1}}, \ldots, v_{d}^{x_{1}}\right)\right)$, for all $x \in U$,

(4) $\mathfrak{m}_{x_{0}}=\left(f_{0}\right)$, for some $f_{0} \in k[X]$,

where $U=X^{*}$, for $X^{*}:=X \backslash\left\{x_{0}\right\}$.

Proof We use basic facts about classical degenerations of algebras and interpretation of classical degenerations in terms of associated $k[X]$-algebras $[4,8]$. It is well known that there exist a nonsingular affine curve $X$, points $x_{0}, x_{1} \in X$, the map $g, k[X]$ algebra $\mathcal{A}$ with suitable $k[X]$-basis, such that conditions (1)-(3) hold. In fact, this is a consequence of a general property of affine varieties with group action: given such action of a connected algebraic group $G$ on affine variety $X$ with open, dense subset $U \subseteq X$ and fixed $x_{0} \in X$ we can always find an affine curve $\Gamma$ with regular map $f: \Gamma \rightarrow X$ such that $f^{-1}(U) \subseteq \Gamma$ is dense in $\Gamma, x_{0} \in f(\Gamma)$ and there exists an element $x \in U$ and a regular map $g: f^{-1}(U) \rightarrow G$ such that $f(y)=g(y) \cdot x$ for all $y \in f^{-1}(U)$ (see for example the discussion on the normal rings in [6] to find how to obtain such a $X, \mathcal{A}$ and $g$ ). Now, to obtain the condition (4), we apply [9, Proposition 2.4]: we replace $X$ by some open neighborhood $V \subseteq X$ of $x_{0}$, and $x_{1}$ by some (any) point $x_{1}^{\prime}$ of the set $U \cap V$.

Recall that we have a natural notion of finite locally bounded $k[X]$-category, which is similar to the notion of finite locally bounded $k$-category. Here the ring $k[X]$ plays a role similar to that of basic field $k$, in particular, homomorphism and radical spaces 
are now free $k[X]$-modules of finite rank (see [4, Definition 2.1] for the precise definition). Using this concept we have the following immediate characterization of idempotent rigid degenerations.

Proposition The algebra $A_{0}$ is an idempotent rigid degeneration of an algebra $A_{1}$ if and only if there exist an irreducible affine variety (even a nonsingular affine curve) $X$ with point $x_{0}$, an open nonempty subset $U \subseteq X$, and a finite locally bounded $k[X]$ category $\mathcal{R}$ satisfying the following conditions:

(1) $A\left(\mathcal{R}^{x_{0}}\right) \cong A_{0}$,

(2) $A\left(\mathcal{R}^{x}\right) \cong A_{1}$, for all $x \in U$.

where $\mathcal{R}^{y}$ is a locally bounded $k$-category with object set ob $\mathcal{R}^{y}:=\mathrm{ob} \mathcal{R}$, and $\mathcal{R}^{y}(a, b):=k[X] / \mathfrak{m}_{x} \otimes_{k[X]} \mathcal{R}(a, b)$ for any $a, b \in$ ob $\mathcal{R}^{y}$.

Proof Apply [4, Lemma 2.2].

\section{4}

We finish this section by formulating a useful lemma, necessary for the proof of the main result.

Lemma Suppose, that $\mathrm{M}=\left[m_{i j}\right] \in \mathbb{M}_{m \times d}(k[X])$ is such that the ranks of all specialized matrices $\mathrm{M}(x):=\left[m_{i j}(x)\right] \in \mathbb{M}_{m \times d}(k)$ do not depend on $x \in X$. Then for any fixed point $x_{0} \in X$ there exist a neighborhood $V \subset X$ of $x_{0}$ and elements $v_{1}, \ldots, v_{d-r} \in$ $(k[V])^{d}$ such that $v_{1}(x), \ldots, v_{d-r}(x)$ form a basis of $\operatorname{ker} \mathrm{M}(x)$ for any $x \in V$, where $r=r\left(\mathrm{M}\left(x_{0}\right)\right)$ is a common rank of matrices $\mathrm{M}(x), x \in X$.

Proof Fix $x_{0} \in X$ and a maximal nonzero minor of a matrix $\mathrm{M}\left(x_{0}\right)$. Without loss of generality we can assume that this minor is realized by rows and columns with indexes $1, \ldots, r$, where $r$ is a common rank of matrices $\mathrm{M}(x), x \in X$. Then we define an open neighborhood $V \subset X$ of $x_{0}$ as the set of all points $x \in X$ such that the $r \times r$-minor of $\mathrm{M}(x)$ corresponding to rows and columns with indexes $1, \ldots, r$ is nonzero. For a such $V$ there exists an invertible matrix $g \in \mathrm{Gl}_{m}(k[V])$ such that matrix $\mathrm{M}^{\prime}:=g \mathrm{M}=\left(m_{i, j}^{\prime}\right)$ has the following form

$$
\left[\begin{array}{rr}
\operatorname{id}_{r} * \\
0 & 0
\end{array}\right]
$$

Then it is easily seen, that for any $x \in V$ the elements of the form

$$
v_{i}:=e_{i+r}-\sum_{j=1}^{r} m_{j, i+r}^{\prime} e_{j},
$$

for $i=1, \ldots, d-r$, determine a bases $v_{1}(x), \ldots, v_{d-r}(x)$ of $\operatorname{ker} \mathrm{M}(x)$. 


\section{Main Result}

\section{1}

For a finite dimensional algebra $A$ we denote by $\mathrm{K}_{0}(A)$ the Grothendieck group of $A$, and by $\operatorname{rk~}_{0}(A)$ its rank. Our aim is to prove the following:

Theorem Let $A_{0}$ and $A_{1}$ be finite dimensional basic algebras of the same dimension. Suppose that $\mathrm{rk} \mathrm{K} 0\left(A_{0}\right)=\operatorname{rkK}_{0}\left(A_{1}\right)$. Then $A_{1} \leq$ cl $A_{0}$ implies $A_{1} \leq_{\text {rig }} A_{0}$.

To prove Theorem 3.1 we need some preparatory facts. One of them is the following general lemma.

Lemma Let $X$ be an affine nonsingular curve, $x_{0} \in X$ a fixed point, and $M$ a torsionfree submodule of a finitely generated, torsion-free $k[X]$-module $N$. Then there exists $V \subset X$ - an open neighborhood of $x_{0}$, such that $M_{V}$ is a free submodule of a free module $N_{V}$.

Proof Apply [9, Proposition 2.4(b)].

\section{2}

From now on we will assume, that $A_{0}, A_{1}$ are basic $d$-dimensional $k$-algebras, where $d \in \mathbb{N}$, such that $A_{1} \leq_{\mathrm{cl}} A_{0}$, and $\operatorname{rk~K} \mathrm{K}_{0}\left(A_{0}\right)=\operatorname{rk~K} \mathrm{K}_{0}\left(A_{1}\right)$, and that $\mathcal{A}$ is as in Theorem 2.2 (we keep whole notation from Theorem 2.2).

Proposition For any ideal $I \triangleleft \mathcal{A}^{x_{1}}$ there exists a unique ideal $\hat{I} \triangleleft \mathcal{A}$, satisfying the following conditions:

(1) $\hat{I}^{x}=g(x) \cdot I \triangleleft \mathcal{A}^{x}$, for all $x \in U$,

(2) $\operatorname{dim}_{k} \hat{I}^{x}=\operatorname{dim}_{k} \hat{I}^{x_{0}}$, for all $x \in X$.

Proof Set

$$
\hat{I}:=\left\{f \in \mathcal{A} ; \forall_{x \in U} f(x) \in g(x) \cdot I\right\} .
$$

Fix $a \in I$. Then the map

$$
f_{a}: U \rightarrow k^{d}
$$

such that $f_{a}(x):=g(x) \cdot a$, for $x \in U$, is a regular morphism, so there exists $m \in \mathbb{N}$ such that the function $\hat{f}_{a}:=f_{0}^{m} f_{a}$ is defined in $x_{0}$. But $\hat{f}_{a} \in \hat{I}$, so (1) holds. Moreover, $\hat{I}$ is an ideal in $\mathcal{A}$, and for $\mathcal{B}:=\mathcal{A} / \hat{I}$ we have $\mathcal{B}^{x} \cong \mathcal{A}^{x_{1}} / I$ for all $x \in U$. Then the torsion part of $\mathcal{B}$ (treated as a $k[X]$-module) consists only of $\mathfrak{m}_{x_{0}}$-torsion elements. Now, due to [9, Lemma 3.2], proving the condition (2) we need only to show that $\mathcal{B}$ is free as a $k[X]$-module, or equivalently, there are no nonzero $\mathfrak{m}_{x_{0}}$-torsion elements in $\mathcal{B}$. 
Let us observe, that $U$ is irreducible as an open subset of an irreducible affine curve, and let $f \in \mathcal{A}$ be such, that coset $\bar{f}$ is $\mathfrak{m}_{x_{0}}$-torsion in $\mathcal{B}$. Then there exists $m \in \mathbb{N}$ and $h \in k[x] \backslash \mathfrak{m}_{x_{0}}$ such that $h f_{0}^{m} f \in \hat{I}$. As a consequence, the subset

$$
Y:=\{x \in U ; f(x) \in g(x) \cdot I\}
$$

includes the nonempty open subset $U \backslash V\left(h f_{0}\right)$, so it is open and nonempty. But the subset

$$
Z:=\{x \in U ; f(x) \notin g(x) \cdot I\}
$$

is also open, $Y \cup Z=U$ and $Y \cap Z=\emptyset$, so by irreducibility of $U$ we infer $Z=\emptyset$, $Y=U$ and $f \in \hat{I}$, hence coset $\bar{f}$ is zero in $\mathcal{B}$.

Now we apply this result for the Jacobson radical.

Lemma Let $I=J\left(\mathcal{A}^{x_{1}}\right)$. Then $\hat{I}^{x_{0}}=J\left(\mathcal{A}^{x_{0}}\right)$.

Proof Clearly $\hat{I}^{x}=J\left(\mathcal{A}^{x}\right)$ satisfies $\left(\hat{I}^{x}\right)^{d}=0$, for every $x \in U$. But the subset

$$
Z:=\left\{x \in X ;\left(\hat{I}^{x}\right)^{d}=0\right\}
$$

is closed in $X$, so $\hat{I}^{x_{0}}$ is nilpotent. Thus $\hat{I}^{x_{0}} \subseteq J\left(\mathcal{A}^{x_{0}}\right)$ and has the same dimension over $k$ as $J\left(\mathcal{A}^{x_{0}}\right)$, hence $\hat{I}^{x_{0}}=J\left(\mathcal{A}^{x_{0}}\right)$.

We set $J:=J(\mathcal{A}), I:=J\left(\mathcal{A}^{x_{1}}\right)$ and $\mathcal{B}:=\mathcal{A} / \hat{I}$.

\section{Corollary}

(a) $\widehat{J\left(\mathcal{A}^{x_{1}}\right)}=J$ and $J$ is nilpotent.

(b) There exists an open neighborhood $V \subseteq X$ of $x_{0}$ such that $\mathcal{B}_{V}=$ $k[V] \otimes_{k[X]}(\mathcal{A} / J)$ is a free $k[V]$-module of rank $n$, where $n=\operatorname{dim}_{k} A_{0} / J\left(A_{0}\right)$.

Proof

(a) All specializations $J^{x}$ are radicals $J\left(\mathcal{A}^{x}\right)$, so are nilpotent in degree $\leq d$, as a consequence, $J$ is nilpotent in degree $\leq d$.

(b) From (a) and Lemma 3.2 we infer that $\mathcal{B}=\mathcal{A} / J$ and $\mathcal{B}$ is torsion-free of rank $n$. Now apply Lemma 3.1.

Now we find (up to some localization of $\mathcal{A}$ by some open neighborhood $V \subseteq X$ of $x_{0}$ ) a complete set of pairwise orthogonal primitive idempotents $e_{1}, \ldots, e_{n} \in \mathcal{A}_{V}$, such that the cosets $\bar{e}_{1}, \ldots, \bar{e}_{n} \in \mathcal{B}_{V}$ form a $k[V]$-base of $\mathcal{B}_{V}$. This is a crucial point in the proof of Theorem 3.1.

The first step realizing this idea is the following

Lemma Let $b_{1}, \ldots, b_{n} \in \mathcal{A}$ be such that $B=\left(\bar{b}_{1}, \ldots, \bar{b}_{n}\right)$ is an ordered base of $\mathcal{B}$. Then there exist an open neighborhood $V \subset X$ of $x_{0}$, which admits the existence of 
an invertible matrix $\mathrm{A} \in \mathbb{M}_{n \times n}(k[V])$ and elements $w_{1}, \ldots, w_{n} \in \mathcal{A}_{V}$ satisfying the following conditions:

(1) the coset $\bar{w}_{i} \in \mathcal{B}_{V}$ of the element $w_{i} \in \mathcal{A}_{V}$ has the coordinate presentation $\bar{w}_{i}^{B}:=$ $\mathrm{A}^{i}$ in base $B_{V}$ of $\mathcal{B}_{V}$ for every $i=1, \ldots, n$, where $\mathrm{A}^{i}$ denotes the $i$-th column of $A$ and $B_{V}$ is the image of $B$ via the standard restriction map,

(2) $w_{i}(x)^{2} \in J^{x}+k \cdot w_{i}(x)$, for all $i=1, \ldots, n$ and $x \in U \cap V$,

(3) $w_{i}(x) w_{j}(x) \in J^{x}$, for all $i, j=1, \ldots, n$ and $x \in U \cap V$.

Proof We fix a $k[X]$-base $E$ of $\mathcal{A}$. Then we can treat elements of $\mathcal{A}$ as column vectors in $\mathbb{M}_{d \times 1}(k[X])$, identifying $a \in \mathcal{A}$ with its coordinate column vector with respect to $E$.

Let $e_{1}^{x_{1}}, \ldots, e_{n}^{x_{1}} \in \mathcal{A}^{x_{1}}$ forms a set of pairwise orthogonal primitive idempotents in $\mathcal{A}^{x_{1}}$, and let $\mathrm{B}=\left[b_{1}|\ldots| b_{n}\right] \in \mathbb{M}_{d \times n}(k[X])$ be the matrix with columns being the vectors $b_{i}$, for $i=1, \ldots, n$. Fix $1 \leq i \leq n$ and set

$$
I_{i}:=J^{x_{1}}+\sum_{j \neq i} k \cdot e_{j}^{x_{1}} .
$$

Then $I_{i}$ is an ideal in $\mathcal{A}^{x_{1}}$ of codimension 1 . As a consequence, $\hat{I}_{i}^{x_{0}}$ is an ideal in $\mathcal{A}^{x_{0}}$ of codimension 1. We find some open neighborhood $V_{i}$ of $x_{0}$ and column matrix $\mathrm{A}^{i} \in \mathbb{M}_{n \times 1}\left(k\left[V_{i}\right]\right)$ such that

$$
\left(\mathrm{B} \cdot \mathrm{A}^{i}\right)(x) \in \bigcap_{l \neq i} \hat{I}_{l}^{x} \backslash\{0\}
$$

for all $x \in V_{i}$. Let $c_{1}, \ldots, c_{s} \in \bigcap_{l \neq i} \hat{I}_{l}$ be a set of generators of the ideal $\bigcap_{l \neq i} \hat{I}_{l}$ treated as a $k[X]$-module, for some $s=s(i)$. Set $\mathrm{D}:=\left[c_{1}|\ldots| c_{s}\right] \in \mathbb{M}_{d \times s}(k[X])$. Let us observe, that all matrices $\mathrm{D}(x)$ and $\mathrm{M}(x):=[\mathrm{B} \mid-\mathrm{D}](x)$, for $x \in X$, have rank $d-n+1$ and $d$ respectively, so inequality $s \geq d-n+1$ holds.

Applying Lemma 2.4 we get an open neighborhood $V_{i}^{\prime}$ of $x_{0}$ and elements:

$$
v_{1}:=\left[\begin{array}{c}
v_{1}^{(b)} \\
v_{1}^{(c)}
\end{array}\right], \ldots, v_{t}:=\left[\begin{array}{c}
v_{t}^{(b)} \\
v_{t}^{(c)}
\end{array}\right] \in k\left[V_{i}^{\prime}\right]^{n+s},
$$

$t=s+n-d$, such that the $v_{1}(x), \ldots, v_{t}(x)$ form a $k$-base of $\operatorname{ker} \mathrm{M}(x)$, for all $x \in V_{i}^{\prime}$. It is clear, that $t \geq 1$, and that we can choose $1 \leq j \leq t$ such that $v_{j}^{(b)}\left(x_{0}\right) \neq 0$. Set $V_{i}:=$ $\left\{x \in V_{i}^{\prime} ; v_{j}^{(b)}(x) \neq 0\right\},\left(\mathrm{A}^{\prime}\right)^{i}:=v_{j}^{(b)}$. Let $\mathrm{A}^{i} \in \mathbb{M}_{n \times 1}\left(k\left[V_{i}\right]\right)$ be the coordinate vector of coset of $\left(\mathrm{A}^{\prime}\right)^{i}$ in $\mathcal{B}$ with respect to the $k[X]$-base $B$ of $\mathcal{B}$. From the equalities $\bigcap_{l \neq i} \hat{I}_{l}^{x}=$ $J^{x}+k \cdot e_{i}^{x}$, for all $x \in U$, we conclude that $w_{i}:=\mathrm{B} \cdot \mathrm{A}^{i} \in \mathcal{A}$ satisfy $w_{i}(x)^{2} \in J^{x}+k$. $w_{i}(x)$, for all $x \in V_{i} \backslash\left\{x_{0}\right\}$. But it is a closed condition, so $w_{i}(x)^{2} \in J^{x}+k \cdot w_{i}(x)$, for all $x \in V_{i}$.

Finally, we set $V:=\bigcap_{i=1}^{n} V_{i}, \mathrm{~A}^{\prime}:=\left[\left(\mathrm{A}^{\prime}\right)^{1}{ }_{\mid V}|\ldots|\left(\mathrm{A}^{\prime}\right)^{n}{ }_{\mid V}\right] \in \mathbb{M}_{d \times n}(k[V])$ and $\mathrm{A}:=$ $\left[\mathrm{A}^{1}{ }_{|V|} \ldots \mid \mathrm{A}_{\mid V}^{n}\right] \in \mathbb{M}_{n \times n}(k[V])$, where $\mathrm{A}_{\mid V}^{i}$ and $\left(\mathrm{A}^{\prime}\right)_{\mid V}^{i}$ are the images of $\mathrm{A}^{i}$ and $\left(\mathrm{A}^{\prime}\right)^{i}$ via the standard restriction map. Clearly, by the construction of $V, \mathrm{~A}^{\prime}$ and $\mathrm{A}$, the cosets of elements $\left(\mathrm{A}^{\prime}\right)^{i}(x), i=1, \ldots, n$, form $k$-bases of $\mathcal{B}^{x}$ for any $x \in V$, so we infer that $\mathrm{A} \in \mathrm{Gl}_{n}(k[V])$. Now it is clear that the proof of the existence of the neighborhood $V$, the matrix A and elements $w_{1}, \ldots, w_{n}$ satisfying the conditions (1)(3) is complete. 
Proposition Let $V$ be as in the lemma. Then there exist $v_{1}, \ldots, v_{n} \in \mathcal{A}_{V}$ such that $\bar{v}_{1}, \ldots, \bar{v}_{n}$ form a base of $\mathcal{B}_{V}$, which consists of pairwise orthogonal primitive idempotents of $\mathcal{B}_{V}$.

Proof We keep the notation from lemma and then set $X:=V, \mathcal{A}:=\mathcal{A}_{V}, \mathcal{B}:=\mathcal{B}_{V}$, $E:=E_{V}$ and so on. Fix $1 \leq i \leq n$. From the orthogonality and linear independence of $\bar{w}_{i}(x)$ 's in $\mathcal{B}^{x}$, for any $x \in X$, we infer that there exists a unique nonzero scalar $\rho_{i}(x) \in k$ such that

$$
\rho_{i}(x) w_{i}(x)-w_{i}^{2}(x) \in \hat{I}_{i}^{x} .
$$

We show that the function $\rho_{i}: X \rightarrow k$ belongs to $k[X]$. It suffices to show that for any $x \in X$ there exists a neighborhood $U_{x}$ of $x$ such that $\rho_{i}$ depends regularly on $y \in U_{x}$.

Fix $x \in X$. Set

$$
\mathrm{B}:=\left[w_{i}\left|w_{i}^{2}\right|-\mathrm{D}\right] \in \mathbb{M}_{n \times(s+2)}(k[V]),
$$

where $\mathrm{D}:=\left[c_{1}|\ldots| c_{s}\right] \in \mathbb{M}_{n \times s}(k[V])$ is formed by a finite set of generators of the radical $J$ (as a $k[X]$-module). It is clear that ranks of all matrices $\mathrm{D}(x)$ and $\mathrm{B}(x)$ are equal to $d-n$ and $d-n+1$, respectively, so $(s+2)-(d-n+1)=s+n-d+1 \geq$ 1. Applying Lemma 2.4 we get an open neighborhood $V_{x} \subseteq X$ of $x$ and elements:

$$
v_{1}=\left[\begin{array}{c}
v_{1}^{(1)} \\
v_{1}^{(2)} \\
v_{1}^{(c)}
\end{array}\right], \ldots, v_{t}=\left[\begin{array}{c}
v_{t}^{(1)} \\
\frac{v_{t}^{(2)}}{v_{t}^{(c)}}
\end{array}\right] \in k\left[V_{x}\right]^{2+s},
$$

where $t:=s+n-d+1$ and $v_{i}^{(c)} \in k[V]^{s}$, for $i=1, \ldots, t$, such that the vectors $v_{1}(y), \ldots, v_{t}(y)$ form $k$-bases of $\operatorname{ker} \mathrm{B}(y)$, for all $y \in V_{x}$. As a consequence of the previous considerations, we infer that $v_{j}^{(1)}(x) \neq 0$ for some $1 \leq j \leq t$. But then, setting $U_{x}:=\left\{y \in V_{x} ; v_{j}^{(1)}(y) \neq 0\right\}$ we get that $v_{j}^{(1)}(y) \cdot w_{i}(y)+v_{j}^{(2)}(y) \cdot w_{i}^{2}(y) \in J^{y}$ for all $y \in U_{x}$. Consequently, $\rho_{i}(y)=-\frac{v_{j}^{(1)}(y)}{v_{j}^{(2)}(y)}$ and $\rho_{i}$ depends regularly on $y \in U_{x}$.

Finally, it is clear, that the inequalities $\rho_{i}(x) \neq 0$, for $x \in X$, imply that $\rho_{i} \in$ $k[X]^{*}$ for every $i=1, \ldots n$. Now the elements $v_{i}:=\rho^{-1} \cdot w_{i}$, for $i=1, \ldots, n$, satisfy our assertion.

\section{4}

In the proof of Theorem 3.1. we will apply also the following general result.

Lemma Let $R$ be a commutative ring, $\mathcal{A}^{\prime}$ a $R$-algebra, $J^{\prime} \triangleleft \mathcal{A}^{\prime}$ a nilpotent ideal for which $\mathcal{A}^{\prime} / J^{\prime}$ is isomorphic to $\prod_{n} R$. Then the standard $R$-base of $\mathcal{A}^{\prime} / J^{\prime}$ consisting of pairwise orthogonal idempotents $\pi\left(\left(v^{\prime}\right)_{1}\right), \ldots, \pi\left(\left(v^{\prime}\right)_{n}\right) \in \mathcal{A}^{\prime} / J^{\prime}$, for some $\left(v^{\prime}\right)_{1}, \ldots,\left(v^{\prime}\right)_{n} \in \mathcal{A}^{\prime}$, can be lifted modulo $J^{\prime}$ to the set of pairwise orthogonal idempotents $\left(e^{\prime}\right)_{1}, \ldots,\left(e^{\prime}\right)_{n} \in \mathcal{A}^{\prime}$, i.e. $\pi\left(\left(e^{\prime}\right)_{i}\right)=\pi\left(\left(v^{\prime}\right)_{i}\right)$, for all $i=1, \ldots, n$, where $\pi: \mathcal{A}^{\prime} \rightarrow$ $\mathcal{A}^{\prime} / J^{\prime}$ is a standard projection.

Proof Apply [1, Proposition 27.1]. 
Proof of Theorem 3.1 Let $V$ and $v_{1}, \ldots, v_{n}$ be as in Proposition 3.3. Then there exists a complete set of pairwise orthogonal idempotents $e_{1}, \ldots, e_{n} \in \mathcal{A}_{V}$ as in Lemma 3.4. Moreover, $\mathcal{B} \cong k[V] \times \ldots \times k[V]$ as a $k[V]$-algebras and the natural epimorphism of $k[V]$-algebra projection $\pi: \mathcal{A}_{V} \rightarrow \mathcal{B}_{V}$ splits. As a consequence, we can assume that $\mathcal{A}_{V}=J_{V} \oplus \mathcal{B}^{\prime}$, where $\mathcal{B}^{\prime}:=\sum_{i=1}^{n} k[V] e_{i} \cong \mathcal{B}_{V}$ as a $k[V]$-algebra.

Define a $k[V]$-category $\mathcal{A}^{b}$ as follows:

$$
\begin{gathered}
\text { ob } \mathcal{A}^{b}=\{1, \ldots, n\}, \\
\mathcal{A}^{b}(i, j):=e_{j} \mathcal{A}_{V} e_{i} .
\end{gathered}
$$

Applying $n^{2}$ times Lemma 3.1, we can assume that we have the splitting

$$
\mathcal{A}^{b}(i, i)=k[V] e_{i} \oplus J\left(\mathcal{A}^{b}(i, i)\right),
$$

where $J\left(\mathcal{A}^{b}(i, i)\right)=e_{i} J e_{i}$ is a free $k[V]$-module of a finite rank and $k[V] e_{i} \cong k[V]$. As a consequence, $\mathcal{A}^{b}$ is a finite locally bounded $k[V]$-category defining idempotent rigid degeneration $A_{1} \leq_{\text {rig }} A_{0}$ (see $[3,4]$ ), and the proof is complete.

Final Remark The author strongly believes, that if we consider the subvariety $Z$ of $\operatorname{alg}_{d}(k)$ consisting of all basic algebras with "dimension vectors isomorphic to $\underline{d}$ ", then the restrictions of $G l_{d}(k)$-orbits to this subvariety are fibre bundles. The difference between $\mathrm{G}_{\underline{d}}(k)$-orbit closure in $\mathrm{lbc}_{\underline{d}}(k)$ and $G l_{d}(k)$-orbit closure in $Z$ seems to have the analogous character to that between the orbit closure for a representations of bounded quiver $(Q, I)$ and the orbit closure in module variety over the algebra $A=$ $k(Q, I)$.

Acknowledgement I would like to thank professor Piotr Dowbor for helpful remarks and comments during the redaction of the paper and the publication process.

Open Access This article is distributed under the terms of the Creative Commons Attribution License which permits any use, distribution, and reproduction in any medium, provided the original author(s) and the source are credited.

\section{References}

1. Anderson, F.W., Fuller, K.R.: Rings and categories of modules. In: Graduate Texts in Mathematics. Springer (1992)

2. Crawley-Boevey, W.W.: Tameness of biserial algebras. Arch. Math. 65, 399-407 (1995)

3. Dowbor, P., Hajduk, A.: A comparison of various types of degenerations for algebras. Algebra Colloq. 14(3), 361-388 (2007)

4. Dowbor, P., Hajduk, A.: Degenerations of covering functors. J. Alg. 357, 138-167 (2012)

5. Gabriel, P.: Finite reprezentation type is open. Representations of algebras. In: Lecture Notes in Math, vol. 488, pp. 132-155. Springer, Berlin (1975)

6. Greuel, G.-M., Laplagne, S., Seelisch, F.: Normalization of Rings. J. Symb. Comput. 45, 887-901 (2010). doi:10.1016/j.jsc.2010.04.002

7. Geiss, Ch.: On degenerations of tame and wild algebras. Arch. Math. 64, 11-16 (1995)

8. Hajduk, A.: CB-degenerations and the rigid degenerations of algebras. Colloq. Math. 106(2), 305-310 (2006)

9. Hajduk, A., Kasjan, S.: On generalized CB-degenerations of algebras. Commun. Algebra, 40(9), 3236-3244 (2012) 
10. Humphreys, J.E.: Linear Algebraic Groups. Springer, New York (1975)

11. Kraft, H.: Geometric methods in representation theory. In: Representations of Algebras, 3rd Int. Conf., Puebla/Mex. 1980, Lect. Notes Math., vol. 944, pp. 180-258 (1982)

12. Mac Lane, S.: Categories for the working mathematician. In: Graduate Texts in Math. 5. Springer (1971)

13. Shafarevich, I.R.: Basic Algebraic Geometry. Springer, Berlin (1994) 